\title{
On the Thermal and Photochemical Stabilities of Photochromic Spirooxazine Dyes Encapsulated in Ormocer Matrices Derived by Sol-Gel Processing
}

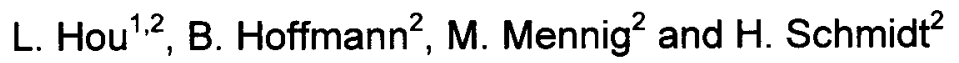 \\ ${ }^{1}$ Shanghai Institute for Optics and Fine Mechanics, Academia Sinica, Shanghai, P.R.China \\ 2 Institut für Neue Materialien, Universitätscampus, Gebäude 43, \\ Im Stadtwald, D-66123 Saarbrücken, Germany
}

Keywords: Spirooxazine-Ormocer, Sol-Gel Processing, Photochromism, Thermal and Photochemical Stabilities

\begin{abstract}
Spiroindolinonaphthoxazine (abbreviated as SO), a most promising class of photochromic dyes due to their better photochromic response to UV irradiation (e.g. sunlight), higher fading rate of the coloured form and higher photofatigue resistance, is incorporated into Ormocer matrices synthesized by the sol-gel route aiming to achieve some application prospects. As for all technical applications the stability of the materials as a function of time and/or under certain harsh circumstances must be considered and improved if necessary. In the present work the thermal and photochemical stabilities of our photochromic SOOrmocer gels and coatings are investigated by room temperature (RT) holding, heat treatment and UV irradiation experiments and compared with the SO-PMMA coatings. It is shown by our results that both the thermal and photochemical stabilities of our SO-Ormocer coatings are similar to those of the SO-PMMA coatings. Considering that both the photochromic response and the fading rate of our SO-Ormocer coatings are much better than those of the SO-PMMA coatings, it is reasonable to conclude that the overall performance of our SO-Ormocer coatings is acceptable for certain application purposes.
\end{abstract}

\section{INTRODUCTION}

There are mainly three most important materials requirements for a photochromic system which consists of photochromic dye and matrix material: high (acceptable) photochromic response to UV irradiation, high or very low fading rate of the coloured form for switching or memory devices, respectively, and high thermal and photochemical stabilities. The last requirement is especially a critical factor to practical applications. Mainly because of unsatisfactory thermal and photochemical stabilities, no large scale markets have been established that commercially exploit photochromic phenomena to date.

The fulfillment of the above requirements depends both upon exploitation of high-performance photochromic dyes and upon development of suitable matrix materials. SO dyes have been shown to possess much higher photochemical stability than spiropyrans (SP) by a factor of $\approx 10^{3}$, while exhibiting similar photochromic response and fading rate to the latter. N. Y. C. Chu [1 5] has published a series of original research papers on the SO-polymer photochromic systems. It turns out from these papers that the photochromic response of the dye is deeply suppressed so that a high dye concentration $(>1 \mathrm{wt} . \%)$ is necessary for obtaining a high enough photochromic response and the fading rate of the colour activated by UV light is largely slackened by a factor of $>10$ as compared to the SO-ethanol solutions so that certain applications which require quick bleaching become impossible

Porous solid materials derived by sol-gel processing, inorganic oxides or organic-inorganic composites (Ormocer), provide new possibilities of hosting photochromic dyes. There are a number of publications dealing with the incorporation of spiropyran (SP) [6 8] and 2,3diphenylindenoneoxide (DPIO) [9] into sol-gel derived matrices, while little has been reported on SO 
dyes. The results concerning SP and DPIO indicate strong matrix-dependence of the photochromism, but no data on the thermal and photochemical stabilities are available. We have been concentrating on SO-Ormocer photochromic systems for 2 years and the results already obtained about the photochromic response and fading rate are very encouraging: The photochromic response levels off during the wetgel-xerogel process and remains almost unchanged up to the present (8 months since preparation) at a satisfactory level with the colour-fading rate being the same as in ethanol [10,11].

The objective of this work is to establish whether the thermal and photochemical stabilities of our SO-Ormocer system are comparable to those of the SO-PMMA system. The SO-Ormocer coatings were subjected to room temperature holding, heat treatment and UV irradiation (suntest) together with the SO-PMMA coatings for comparison. The photochromic intensity $\left(\Delta \mathrm{A}_{0}\right)$ and halflife time $\left(\mathrm{t}_{0.5}\right)$ of the coloured form were monitored during these treatment processes. The results are very positive: the SO-Ormocer coatings exhibit similar thermal and photochemical stabilities to the SO-PMMA coatings while their photochromic response and fading rate are much more favourable to applications in switching devices than the latter.

\section{EXPERIMENTAL}

The preparation procedure of the SO-Ormocer gels and coatings is depicted in Fig. 1. Appropriate amounts of methyltrimethoxysilane (MTMS) and glydidyloxytrimethoxysilane (GPTMS) and water in the form of $0.1 \mathrm{~N} \mathrm{HNO}_{3}$ solution were first mixed by magnetic stirring for ten minutes in a plastic container $(\varnothing 30 \times 70 \mathrm{~mm})$ and then subjected to $20 \mathrm{kHz}$ ultrasonic radiation at a power of $20 \mathrm{~W}$ for 20 minutes. The ultrasonic wave was supplied by a SONIFIER Model 450 device (Branson Ultrasonics CT) through a standard horn tip immersed in the liquid mixture. The sols were then cooled down to room temperature, followed by the addition of appropriate amounts of additives and the introduction of dye-ethanol solution. After a $1 \mathrm{~h}$-magnetic stirring the sols were exposed to the open air with continuing magnetic stirring. When a final volume of $20 \mathrm{ml}$ was reached the sols became viscous and the containers were then closed

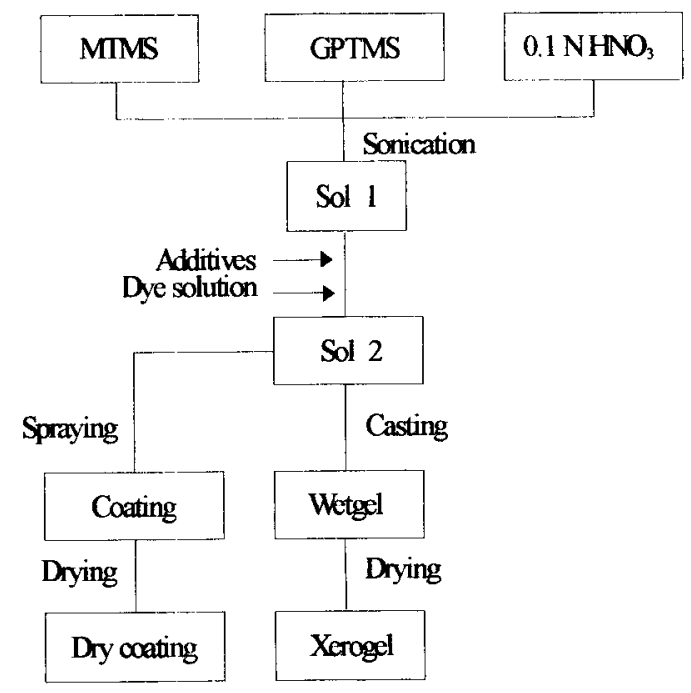

Fig. 1: Preparation procedure of SO-doped Ormocer gels and coationg

Samples used for monitoring the evolution of the photochromism during RT-holding were prepared by pouring the viscous sols into polystyrene cell of $4 \mathrm{~mm}$ thick and keeping them open to the atmosphere. Coatings of various thickness for thermal (heat treatment) and photochemical (UV 
irradiation) stability tests were produced by spraying appropriate volumes of the viscous sols on slide glass substrates.

The aluminosilicate samples were derived from di-iso-butoxy-aluminoxy-triethoxysilane (ASE) using iso-propanol (i-PrOH) as a solvent. At first, the $\mathrm{H}_{2} \mathrm{O}-\mathrm{i}-\mathrm{PrOH}$ mixture was added to the ASE-i$\mathrm{PrOH}$ mixture under vigorous magnetic stirring for $\mathrm{lh}$. Then the dye-ethanol solution was introduced slowly under vigorous stirring, too. After an additional $1 \mathrm{~h}$-stirring the resultant sols were transferred into polystyrene cells $(10 \times 10 \times 45 \mathrm{~mm})$ with stoppers and left to gel and age.

For the purpose of comparison, SO-PMMA coatings were also prepared by spraying on slide glass substrates the SO-PMMA-EA (ethylacetate) solution which contains $15 \mathrm{~g}$ PMMA in $100 \mathrm{ml}$ ethylacetate

Fig. 2 shows schematically the apparatus used for the real-time measurement of photochromism. UV irradiation is from a Hg-lamp and the Schott UV-pass filter UG5 is used to eliminate the influence of heat on the colouring-fading process. From the recorded transmission vs. time curves, photochromic intensity $\left(\Delta \mathrm{A}_{0}\right)$ and half-life time $\left(\mathrm{t}_{0.5}\right)$ are evaluated. Their variations during $\mathrm{RT}$ holding or after heat treatment at different temperatures and after UV irradiation for different lengths of time are used as a measure of the corresponding stabilities.

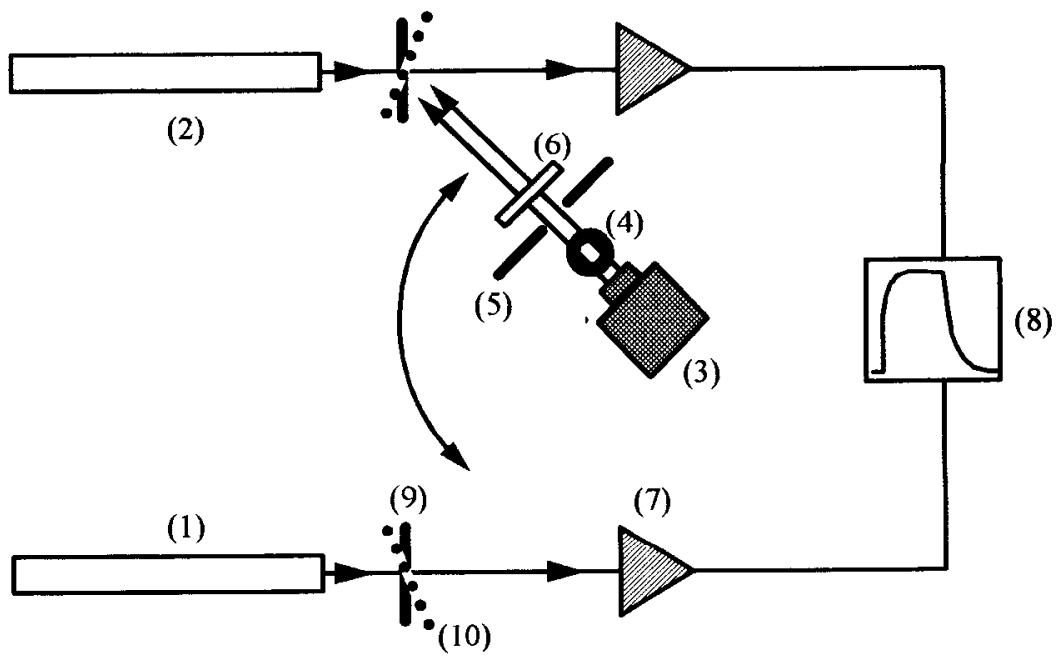

Fig.2: Schematic layout of the apparatus used for colouring-fading measurement
(1) green He-Ne-laser
(2) red He-Ne-laser
(3) Hg-lamp
(4) Focussing system
(5) Shutter
(6) UV-pass filter
(7) Detector
(8) Recorder
(9) Cell sample
(10) Coating sample

8)

A SUNTEST CPS (Heraeus) apparatus was employed to characterize the photochemical stability of the samples against UV irradiation at an intensity of $600 \mathrm{~W} / \mathrm{cm}^{2}$. Heat treatments were conducted in a MEMMERT model 100 electric furnace.

\section{RESULTS AND DISCUSSION}

\section{Stability at room temperature}

Fig. 3 demonstrates the severe degradation of the photochromic response of SO in ASE-gels during ageing. It is evident that the photochromic intensity decreases by a factor of $\approx 3$ after 14 days and vanishes after 60 days ageing at room temperature. It is well known that SO dyes undergo ringopening reactions under UV irradiation. During the ageing process of the ASE-gels the structural change of the SO-molecules would face greater and greater hindrance due to the increase of 
condensation extent of the Al-O-Si network, therefore, the photochromic transformation is more and more heavily suppressed and finally nullified. Moreover, certain chemical bonding between $\mathrm{Al}$ in the matrix and $\mathrm{O}$ in the dye molecules may also be responsible at least partly for the degradation of the photochromic response.

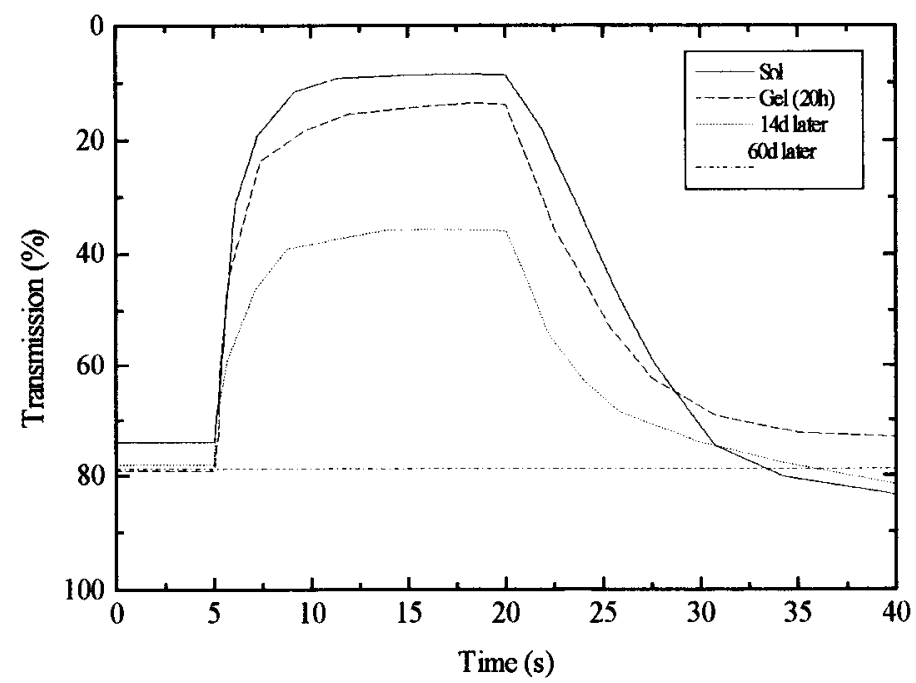

Fig.3: Colouring-fading curves of SO $\left(C=1 \times 10^{-4} \mathrm{~mol} / \mathrm{l}\right)$ in ASE-gel at different time after preparation (gelling time $=20 \mathrm{~h}$ )

The RT-holding stability of SO is greatly improved by using Ormocer as matrix materials. This is clearly indicated by Fig. 4. Although $\Delta \mathrm{A}_{0}$ of the gel decreases at the early stages of the sol-gel transformation, it levels off in about one month after preparation.

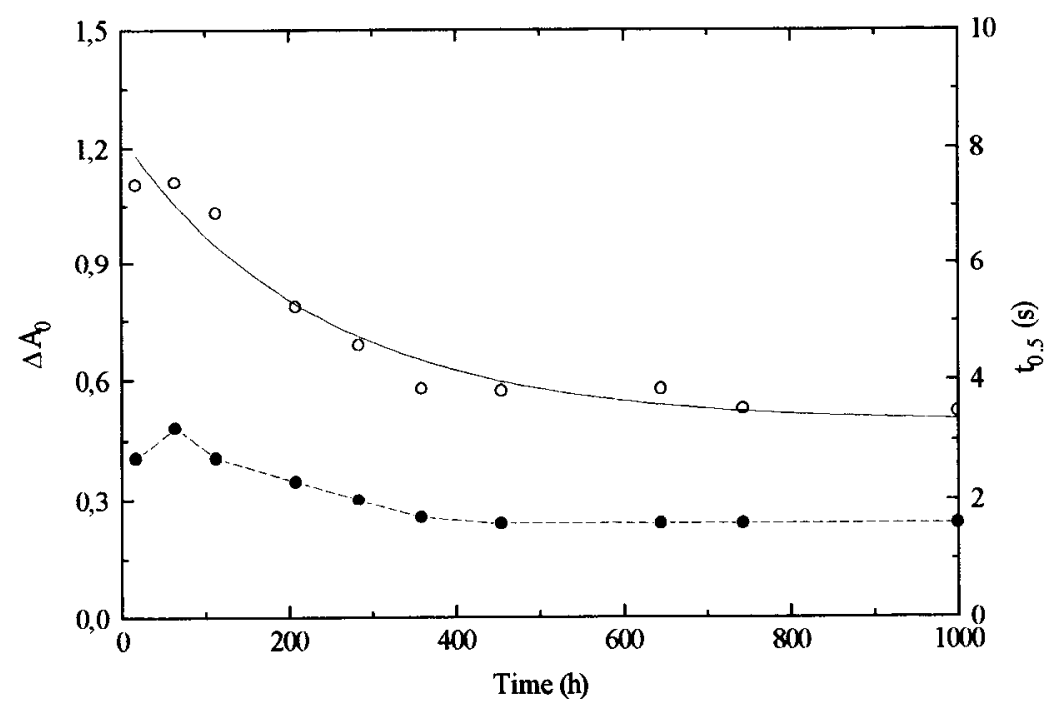

Fig.4: Variation of photochromic intensity $\left(\Delta \mathrm{A}_{0}\right)$ and half-life time $\left(\mathrm{t}_{0.5}\right)$ of $\mathrm{SO}\left(2.5 \times 10^{-3} \mathrm{~mol} / \mathrm{l}\right)$ in Ormocer gel during sol-gel transformation 
Furthermore, the $\Delta \mathrm{A}_{0}$ remains almost constant (ca. 0.60 ) up to the present (6 months after preparation). Another interesting result is that to.5 increases in the early stage (within $100 \mathrm{~h}$ after preparation), then decreases and finally levels off in about one month, too. These results can be reasoned in terms of the viscosity increase before gelling and the difference in degree of freedom of the dye molecules entrapped in the pores and out of the pores. With the increase in the viscosity of the sol due to the progressive condensation, a greater and greater portion of dye molecules are more and more tightly confined so that the possibility of photochormic transformation decreases gradually in a statistical sense. Meanwhile, the fading speed of the coloured form decreases due to the increasing hindrance. At the gelling point, a certain portion of the dye molecules has been "killed" and loses its photochromic activity. Only those dye molecules caged in the pores in the gel matrix remain photochromically active and these molecules are completely free within the cages as in ethanol solution. Therefore, in the xerogel matrix both $\Delta \mathrm{A}_{0}$ and $\mathrm{t}_{0.5}$ reach a steady value, $\Delta \mathrm{A}_{0}$ depends upon matrix composition while $t_{0.5}$ is equal to the value in ethanol solution $(\approx 1.5 \mathrm{~s})$ independent of matrix composition.

\section{Stability against heat treatment}

The variation of $t_{0.5}$ and relative loss of $\Delta A_{0}$ of the SO-Ormocer coating with heat treatment temperature is shown in Fig. 5, from which it can be seen that heat treatment has little influence on $t_{0.5}$ up to $120^{\circ} \mathrm{C}$ and leads to limited increase in the relative loss of $\Delta \mathrm{A}_{0}$, ca $10 \%$ up to $120^{\circ} \mathrm{C}$. This result implies the possibility of curing the SO-Ormocer mterials thermally at around $120^{\circ} \mathrm{C}$ without significant degradation of the photochromic performance as heat treatment is generally necessary in order to achieve desired flexibility (or rigidity) of the Ormocer matrices hosting photochromic dyes because the flexibility of the matrices is a conflicting factor which affects photochromic response and photochemical stability in opposite directions: higher flexibility is always favourable to the former but unfavourable to the latter.

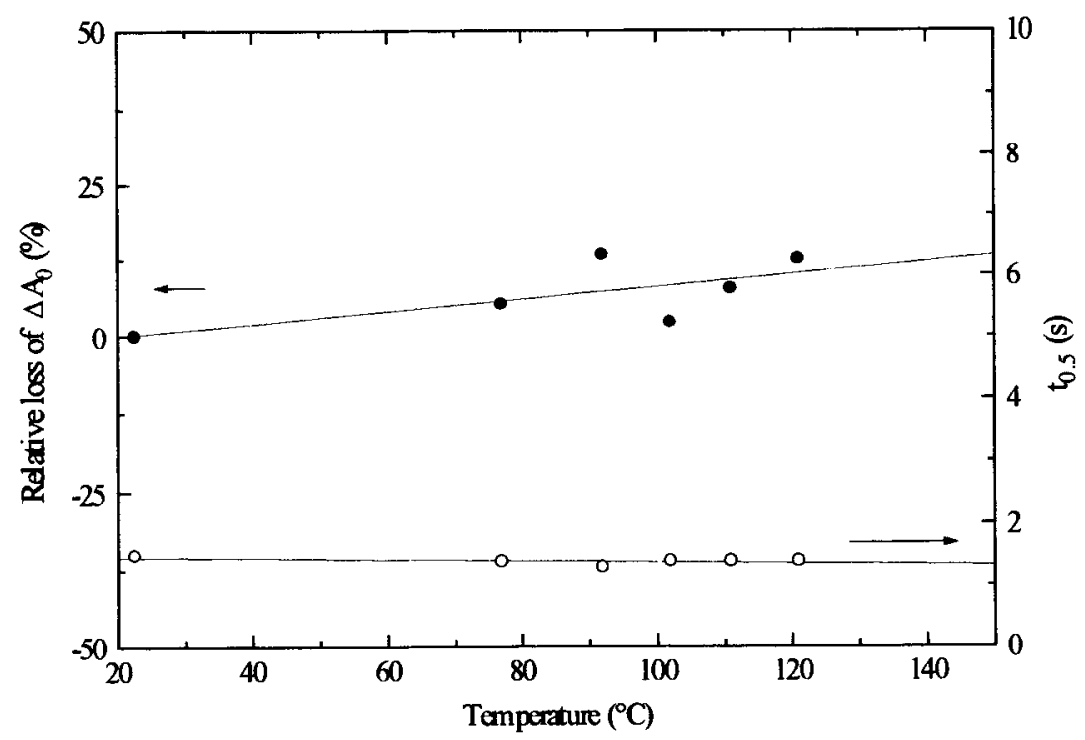

Fig. 5: to.5 and relative loss of $\Delta \mathrm{A}_{0}$ of $\mathrm{SO}\left(2.5 \times 10^{-3} \mathrm{~mol} / \mathrm{l}\right)$-Ormocer coating as a function of heat treatment temperature

Fig. 6 presents the variation of $\triangle \mathrm{A}_{0}$ and $\mathrm{t}_{0.5}$ of SO-PMMA coatings with $\mathrm{RT}$-holding time and heat treatment. It follows from this figure that $\triangle \mathrm{A}_{0}$ shows no significant decrease below $120^{\circ} \mathrm{C}$ 
while heat treatment at $140^{\circ} \mathrm{C}$ for $6 \mathrm{~h}$ results in a relative loss of $\Delta \mathrm{A}_{0}$ less than $25 \%$ and the peelingoff of the coating. However, $\mathrm{t}_{0.5}$ increases very rapidly with increasing heat treatment temperature, and even during RT-holding it increases slowly, too. Based on the results from Figs. 5 and 6 one may conclude that the thermal stability of $\triangle A_{0}$ of the SO-PMMA system is a little better than the SOOrmocer system whereas the thermal stability of $\mathrm{t}_{0.5}$ of the SO-Ormocer system is much better than the SO-PMMA system. Or one can say that the thermal stability of the overall photochromic performance of the SO-Ormocer and SO-PMMA systems is similar.

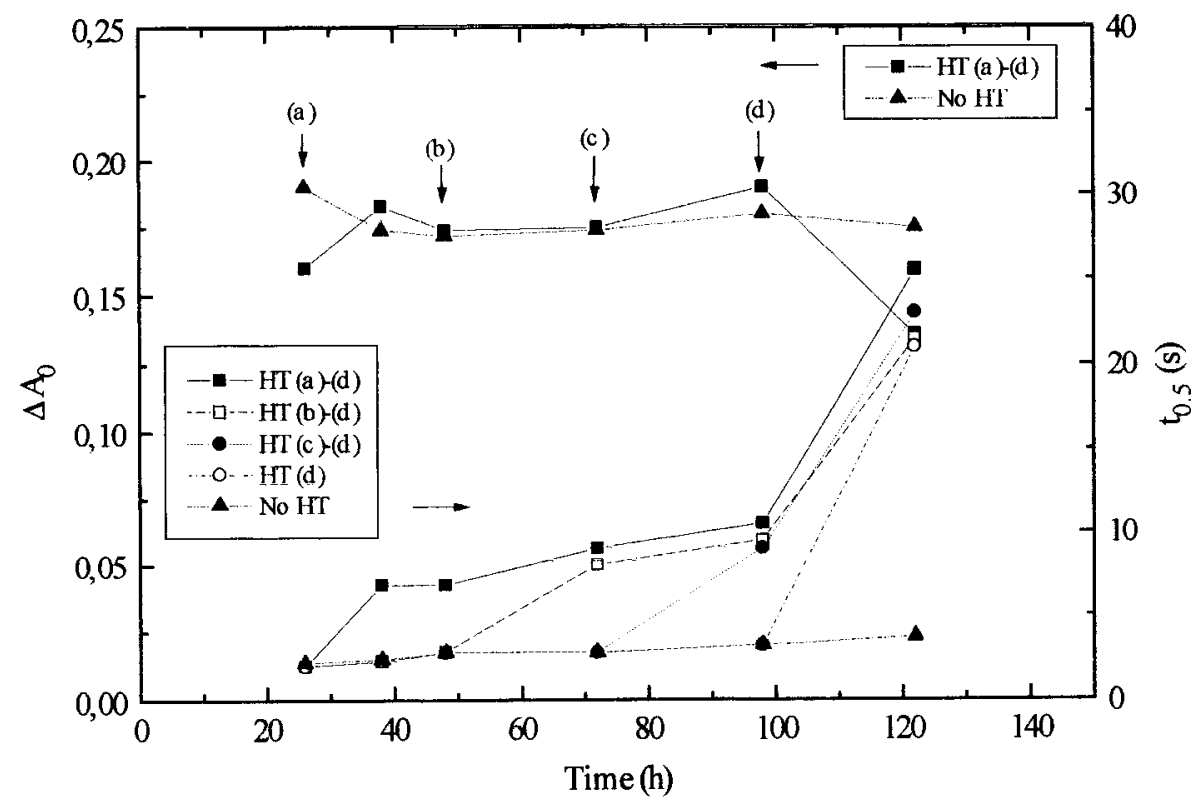

Fig.6: Variation of $\mathrm{t}_{0.5}$ and $\triangle \mathrm{A}_{0}$ of $\mathrm{SO}\left(1 \times 10^{-3} \mathrm{~mol} / \mathrm{l}\right)$-PMMA coatings with RT-holding time and heat treatment (HT): (a) $100^{\circ} \mathrm{C} / 12 \mathrm{~h}$, (b) $110^{\circ} \mathrm{C} / 6 \mathrm{~h}$, (c) $120^{\circ} \mathrm{C} / 6 \mathrm{~h}$, (d) $140^{\circ} \mathrm{C} / 6 \mathrm{~h}$

\section{Photochemical stability against UV irradiation}

Another conflicting factor to a photochromic material is the exposure to UV light. UV irradiation is indespensable to photochromism but harmful to the dye molecules. This is why solid matrices are preferred for photochromic dyes. The utilization of polymer matrices leads to significant enhancement of photochemical stability but at the same time poses a deep supression on both the photochromic response and the colour-fading rate. It has already been established that both the photochromic response and the colour-fading rate of SO-Ormocer gels and coatings are much higher than the SO-PMMA coatings. However, a comparison in the photochemical stability between the two materials must be made in order to confirm the feasability of practical applications of the SOOrmocer material. Fig. 7 gives a comparison between SO-ethanol, SO-Ormocer and SO-PMMA. It turns out from this figure that (1) the photochemical stability of SO-Ormocer coating without any additives is between the SO-ethanol and SO-PMMA systems. (2) A small amount of an additive leads to a great improvement of the photochemical stability of SO-Ormocer coating, becoming considerably better than the SO-PMMA coating with the same SO concentration. (3) By increasing concentrations of SO in the SO-PMMA systems to $5 \times 10^{-3} \mathrm{~mol} / 1$ the photochemical stability is further enhanced. This suggests more elaborate research efforts to be made to further improve the 
photochemical stability of our SO-Ormocer materials. Increasing SO concentration and introducing more effective additives may be helpful forthcoming steps.

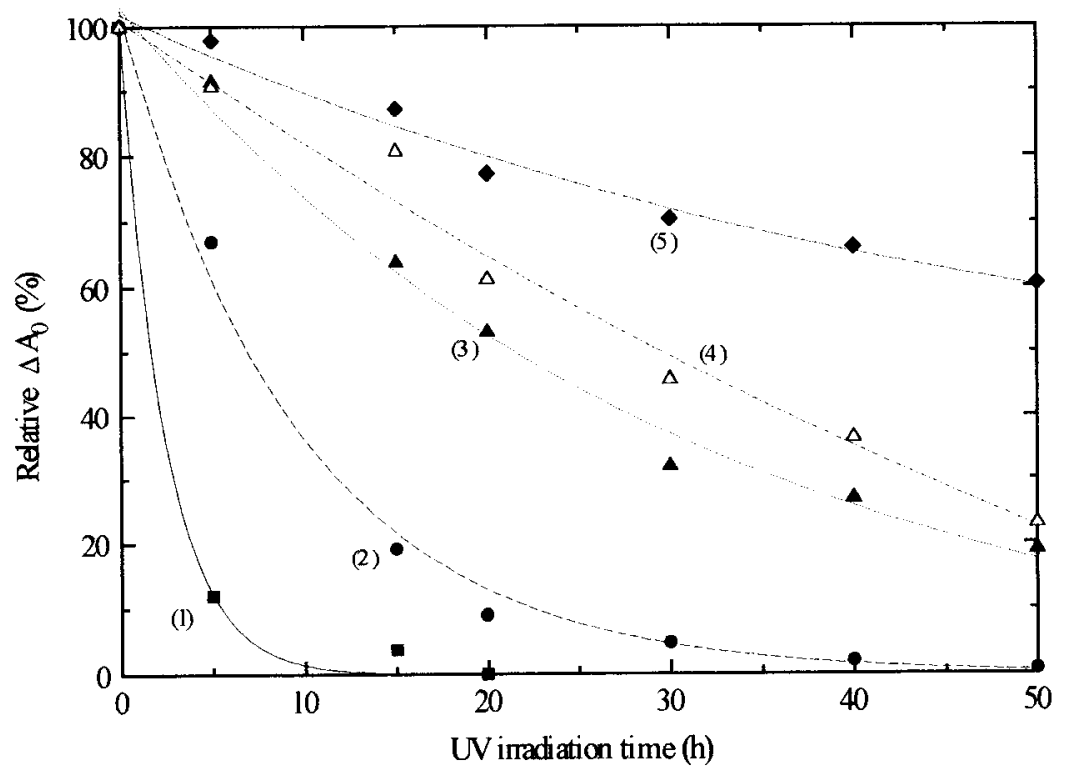

Fig.7: Decrease of $\Delta \mathrm{A}_{0}$ of SO with UV irradiation time in different matrices: (1) Ethanol (C = $\left.4 \times 10^{-3} \mathrm{~mol} / \mathrm{l}\right)$, (2) Ormocer coating $\left(\mathrm{C}=1 \times 10^{-3} \mathrm{~mol} / \mathrm{l}\right)$ without additives, (3) PMMA coating $\left(\mathrm{C}=1 \times 10^{-3} \mathrm{~mol} / \mathrm{l}\right),(4)$ Ormocer coating $\left(\mathrm{C}=1 \times 10^{-3} \mathrm{~mol} / \mathrm{l}\right)$ with an additive, (5) PMMA coating $\left(\mathrm{C}=5 \times 10^{-3} \mathrm{~mol} / \mathrm{l}\right)$

\section{CONCLUSION}

We are now in a position to tailor photochromic SO-Ormocer materials with the thermal and photochemical stabilities being comparable to, and both the photochromic response and the colourfading rate much better than the SO-PMMA materials.

\section{REFERENCES}

[1] N. Y. C. Chu, SPIE's 29th Annual International technology Symposium Optical and Electrooptical Engineering 562-03, 6 (1985)

[2] N. Y. C. Chu, Solar Energy Materials 14, 215 (1986)

[3] N. Y. C. Chu, Optical Materials Technology for Energy Efficiency and Solar Energy Conversion VII, SPIE Vol. 1016, 152 (1988)

[4] N. Y. C. Chu, Energy Res. Abstr. 14(10), No. 20059 (1989)

[5] N. Y. C. Chu, SPIE Institute Series IS4, 102 (1990)

[6] D. Levy and D. Avnir, J. Non-Cryst. Solids 113, 137 (1989)

[7] D. Preston, J.-C. Pouxviel, T. Novinson et al., J. Phys. Chem. 94, 4167 (1990)

[8] J. I. Zink and B. Dunn, J. Mater. Chem. 1, 903 (1991)

[9] S. A. Yamanaka, J. I. Zink and B. Dunn, Proc. SPIE 1758, 372 (1992)

[10] L. Hou, B. Hoffmann, M. Mennig and H. Schmidt, J. Sol-gel Sci. Techn. (in press)

[11] L. Hou, M. Mennig and H. Schmidt, Proceedings of the International Symposium on Glass Science and Technology, Oct. 6 8, 1993, Athens, Greece (in press) 